\title{
Engineered Ammonia Lyases for the Production of Challenging Electron-rich L-Phenylalanines
}

\author{
Syed T. Ahmed, Fabio Parmeggiani, Nicholas J. Weise, Sabine L. Flitsch, and Nicholas J. Turner*
}

Manchester Institute of Biotechnology (MIB), School of Chemistry, University of Manchester, 131 Princess Street, M1 ${ }_{7} \mathrm{DN}$, Manchester, United Kingdom

\begin{abstract}
Engineered variants of phenylalanine ammonia lyase from Planctomyces brasiliensis were developed through rational design efforts focusing on the aryl binding pocket of the active site, guided by structural and phylogenetic inference. Inherent problems traditionally associated with the biocatalytic hydroamination of acrylic acids, such as low conversion and poor regioselectivity with alkyl and methoxy derivatives, could be overcome. The PbPAL variants described here represent a valuable addition to the biocatalytic toolbox, allowing previously inaccessible amino acid building blocks to be obtained regio- and enantioselectively on preparative scale.
\end{abstract}

KEYWORDS: biocatalysis; protein engineering; amino acids; ammonia lyases; industrial biotechnology

While the synthetic utility of phenylalanine ammonia lyase (PAL) enzymes ${ }^{1}$ has been demonstrated by the biocatalytic hydroamination of a broad range of substituted cinnamic acids ${ }^{2}$ and also in chemo-enzymatic syntheses, ${ }^{3}$ the substrate scope of PALs has been limited primarily to compounds with electron-poor/neutral ring systems, with no general success in engineering PALs to convert efficiently electron-rich analogues (Scheme ia).

Recently, we reported on the identification of several new PALs, including those from Dictyostelium discoideum (DdPAL) and Planctomyces brasiliensis (PbPAL), exhibiting variable activity profiles towards a broad panel of simple cinnamic acids. ${ }^{4}$ Remarkably, both PbPAL and DdPAL were able to convert $m$-methoxycinnamic acid (a substrate shown to be converted poorly, if at all, by other PALs) to the corresponding amino acid. While the active site residues are nearly identical, huge differences in conversion were observed between the enzymes, demonstrating the contribution of amino acid residues distal to the active site. Herein, we describe our engineering efforts to generate a panel of PAL biocatalysts with an expanded substrate scope, able to accept a broad range of demanding electron-rich substrates.

A panel of cinnamic acids bearing electron-donating substituents (Scheme 1b), synthesised from the corresponding aldehydes by a Knoevenagel-Doebner condensation (Supporting Information), was tested with several PALs, in the form of whole cells of recombinant E. coli overproducing the enzyme, and ammonium

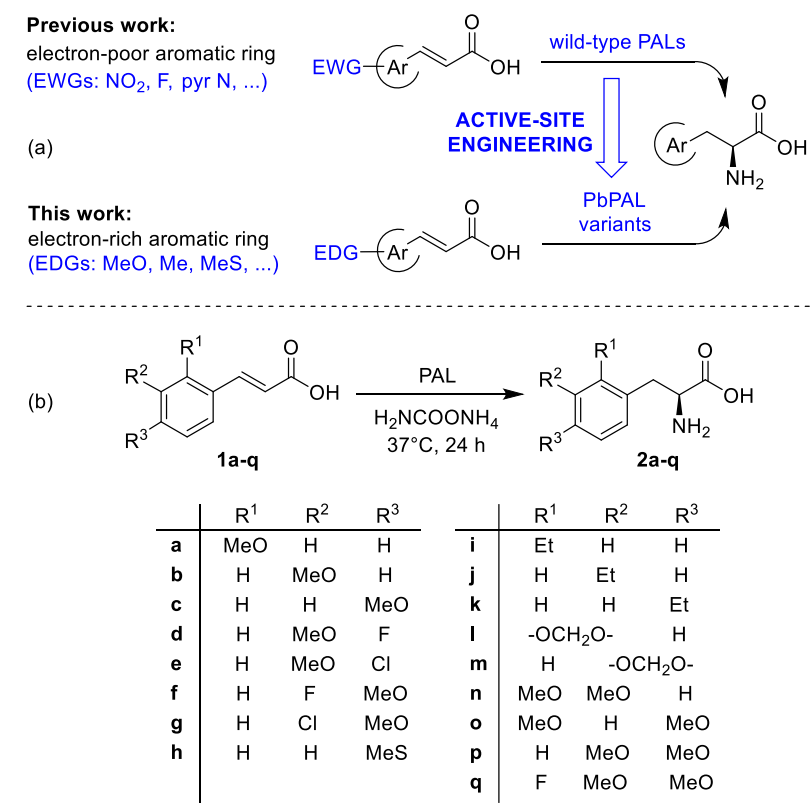

Scheme 1. Lack of suitable PAL catalysts for electron-rich phenylalanines (a). Panel of challenging electron-rich cinnamic acid substrates (1a-q) selected for this study (b).

carbamate as the ammonia source (Figure 1). PbPAL and DdPAL were tested, along with the well-known biocatalysts AvPAL from Anabaena variabilis and RgPAL from Rhodotorula glutinis for comparison (Figure 1). Of the four enzymes, PbPAL was the only one to give 


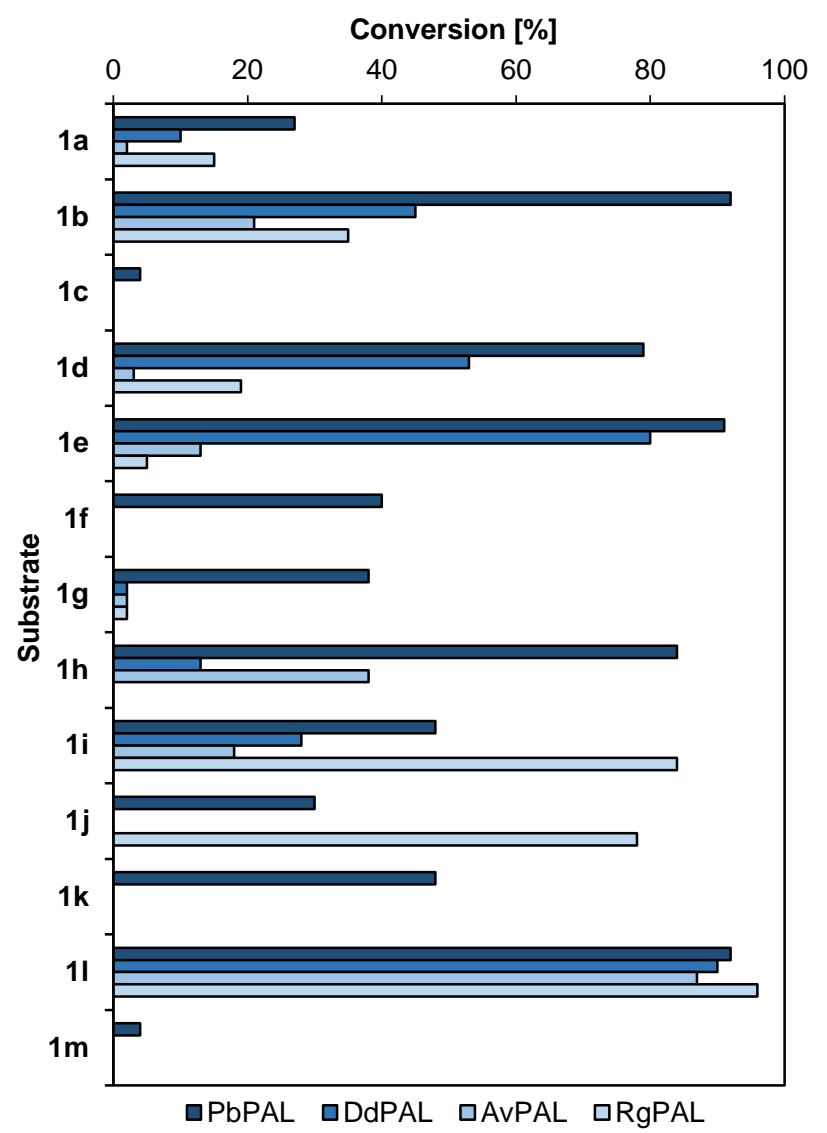

Figure 1. Screening of four different wild-type PALs against electron-rich substrates (substrates $\mathbf{1 n}-\mathbf{q}$ are omitted since they did not show any conversion with any of the tested enzymes). Expt. cond.: $5 \mathrm{mM}$ 1a-q, $4 \mathrm{M}$ $\mathrm{H}_{2} \mathrm{NCOONH}_{4}$, $50 \mathrm{mg} \mathrm{mL}{ }^{-1}$ E. coli (PAL), $\mathrm{pH} \sim 10$ (unadjusted), $37^{\circ} \mathrm{C}, 24 \mathrm{~h}$.

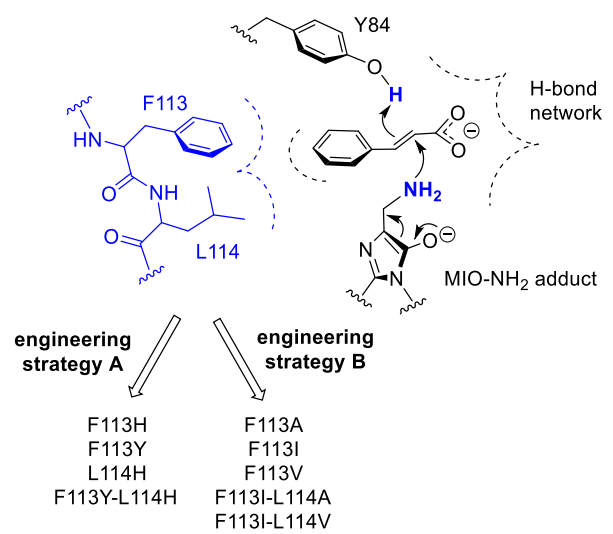

Figure 2. Schematic representation of the binding site of PbPAL, highlighting the positions selected for engineering.

moderate to good conversion of substrates $\mathbf{1 a}-\mathbf{b}$ and $\mathbf{1 d -}$ 1, even though very low or no activity was displayed with compounds $\mathbf{1 c}$ and $\mathbf{1 m - q}$. It is worth noting that three of the substrates $(\mathbf{1} \mathbf{b}, \mathbf{i}, \mathbf{l})$ were seen to afford the corresponding amino acids in high conversion with several PALs.
This interesting observation shows that the poor conversion of most substrates in this panel should not be ascribed only to the presence of electron-donating substituents, but rather to a complex interplay of stereoelectronic factors and affinity to the enzyme active site.

Nevertheless, in order to increase the conversions of the poorer substrates, PbPAL was selected as a promising starting point and two separate engineering strategies were employed to broaden its substrate range. We targeted two key residues in the aryl binding pocket of the active site of PbPAL (F113 and L114, or FL, based on sequence alignment), also known as "selectivity residues" because they confer to the enzyme its substrate preference for either phenylalanine, tyrosine or histidine (PAL, TAL, HAL, respectively). ${ }^{1 a}$ The first of these engineering approaches (Figure 2, strategy A) was the replacement of the two residues with those of other members of the enzyme family known to bind hydroxylated amino acids such as Ltyrosine and L-DOPA., ${ }^{5}$ Combinations of $\mathrm{HL}^{5} \mathrm{YH}^{6}$ and $\mathrm{YL}^{7}$ were seen, as compared with the homologous FL in PbPAL (and most PALs), therefore we chose to investigate variants $\mathrm{F}_{113} \mathrm{H}, \mathrm{F}_{113} \mathrm{Y}$ and $\mathrm{L}_{114} \mathrm{H}$. The second approach (Figure 2, strategy B) was based on converting these residues to smaller but still hydrophobic amino acids to widen the active site cavity around the para-position of the substrate, as attempted in previous studies. ${ }^{3,8,9}$ For this design strategy we selected variants F113A, F113I and F113V. All mutations were introduced by site-directed mutagenesis and the variants produced in $E$. coli for activity tests under the same conditions used for the WT enzyme (Table 1). No appreciable differences in the expression levels were observed compared to WT, with the only exception of $\mathrm{L}_{11} \mathrm{H}$ that showed considerably lower soluble expression (Supporting Information, Figure $\mathrm{S}_{1}$ ).

Rewardingly, considerable improvements were seen with almost all the substrates considered, with the only exception of $\mathbf{1 b}$ (already highly converted by WT) and $\mathbf{n i}$. The most significant increases in conversion were observed with meta- and para- substituted compounds, and in particular substrates $\mathbf{1 c}, \mathbf{f}, \mathbf{g}, \mathbf{h}, \mathbf{m}$ (all bearing a heteroatom at the para-position) were better accepted by all the variants tested.

As a general trend, the widened active site variants (strategy B) performed better than the TAL-like variants (strategy A). A notable exception is $\mathbf{1 d}$, for which both the TAL-like variants gave better yields (presumably due to hydrogen bonding between the $\mathrm{Y} / \mathrm{H}$ residue and the fluorine atom). Remarkably, significant improvements in conversion of the highly electron-rich substrates $\mathbf{1 m - q}$ were achieved (e.g., >12-fold for $\mathbf{1 m}$ with F113I), including the creation of new activity for $\mathbf{1 n}-\mathbf{q}$, not converted by the WT enzyme, but accepted by some of the variants, albeit with low conversions (3-12\%).

In an effort to improve conversions further, we also investigated double variants: F113Y-L114H (for strategy A), F113I-L114A and F113I-L114V (for strategy B). As observed with L114H, also F113Y-L114H showed lower soluble 
Table 1. Biotransformation of electron-rich cinnamic acids by PbPAL variants. ${ }^{a}$

\begin{tabular}{|c|c|c|c|c|c|c|c|c|c|c|}
\hline \multirow[b]{2}{*}{ Subs. ${ }^{b}$} & \multirow[b]{2}{*}{$\mathbf{R}$} & \multirow[b]{2}{*}{$\begin{array}{c}\text { WT } \\
\text { Conv. } \\
{[\%]^{\mathrm{c}}}\end{array}$} & \multicolumn{2}{|c|}{ Strategy A variants } & \multicolumn{5}{|c|}{ Strategy B variants } & \multirow[b]{2}{*}{$\begin{array}{c}\text { Fin3Y-L114I } \\
\text { Conv. } \\
{[\%]^{\mathrm{c}}} \\
\end{array}$} \\
\hline & & & $\begin{array}{c}\text { Fins } \\
\text { Conv. } \\
{[\%]^{c}} \\
\end{array}$ & $\begin{array}{c}\text { Fi13Y } \\
\text { Conv. } \\
{[\%]^{c}} \\
\end{array}$ & $\begin{array}{c}\text { F113A } \\
\text { Conv. } \\
{[\%]^{c}} \\
\end{array}$ & $\begin{array}{c}\text { FinI I } \\
\text { Conv. } \\
{[\%]^{c}} \\
\end{array}$ & $\begin{array}{c}\text { F113V } \\
\text { Conv. } \\
{[\%]^{c}} \\
\end{array}$ & $\begin{array}{c}\text { F113I-L114A } \\
\text { Conv. } \\
{[\%]^{c}} \\
\end{array}$ & $\begin{array}{c}\text { F113I-L114V } \\
\text { Conv. } \\
{[\%]^{c}}\end{array}$ & \\
\hline 1a & $2-\mathrm{MeO}$ & 27 & 7 & 22 & 30 & 8 & 9 & - & - & 4 \\
\hline $\mathbf{l b}$ & 3-MeO & 92 & 55 & 91 & 89 & 92 & 89 & 4 & 92 & 76 \\
\hline Ic & $4-\mathrm{MeO}$ & 8 & 55 & 60 & 40 & 67 & 30 & 3 & 44 & 48 \\
\hline 1d & $4-\mathrm{F}-3-\mathrm{MeO}$ & 79 & 91 & 90 & 67 & 30 & 26 & - & 24 & 88 \\
\hline If & $3-\mathrm{F}-4-\mathrm{MeO}$ & 40 & 86 & 87 & 87 & 87 & 86 & - & 86 & 87 \\
\hline 19 & $3-\mathrm{Cl}-4-\mathrm{MeO}$ & $3^{8}$ & 78 & 42 & 70 & 88 & 77 & 8 & 84 & 73 \\
\hline $\mathbf{l h}$ & 4-MeS & 84 & 95 & 94 & 95 & 95 & 96 & 87 & 95 & 94 \\
\hline li & 2 -Et & 48 & 9 & 24 & 24 & 11 & 8 & - & - & 26 \\
\hline $\mathbf{i j}$ & $3-\mathrm{Et}$ & 30 & 78 & - & 86 & 80 & 88 & 24 & 85 & 4 \\
\hline $\mathbf{~} \mathbf{k}$ & 4 -Et & 48 & - & 17 & 84 & 75 & 85 & 14 & 84 & 17 \\
\hline $1 \mathrm{~m}$ & $3,4-\mathrm{OCH}_{2} \mathrm{O}$ & 3 & 42 & 27 & 54 & 67 & 48 & - & 48 & 11 \\
\hline in & $2,3-(\mathrm{MeO})_{2}$ & - & 3 & - & 2 & 10 & - & - & 2 & - \\
\hline 10 & $2,4-(\mathrm{MeO})_{2}$ & - & - & - & - & 6 & - & - & - & - \\
\hline 1p & $3,4-(\mathrm{MeO})_{2}$ & - & 4 & 12 & - & - & - & - & - & 18 \\
\hline 19 & $2-\mathrm{F}-3,4-\mathrm{MeO}$ & - & 3 & 9 & - & - & - & 3 & - & - \\
\hline
\end{tabular}

${ }^{\text {a }}$ Expt. cond.: $5 \mathrm{mM}$ ra-q, $4 \mathrm{M} \mathrm{H}_{2} \mathrm{NCOONH}_{4}, 50 \mathrm{mg} \mathrm{mL}^{-1}$ E. coli (PAL), $\mathrm{pH} \sim 10$ (unadjusted), $37^{\circ} \mathrm{C}, 24 \mathrm{~h}^{\mathrm{b}}$ Substrates re and $1 \mathbf{l}$ are omitted since they afforded good conversions with the WT enzyme (91 and 92\%, respectively). ${ }^{c}$ Determined by HPLC. The highest conversion obtained for each substrate is highlighted.

expression levels (Figure $\mathrm{S}$ ). Biotransformation results are reported in Table 1. The switching of L114 to smaller hydrophobic residues in the F113I variants did not afford any improvement for the substrates tested, and was even detrimental in some instances (e.g. 1a,c,i).

An attempt to combine the two strategies led us to modify further the best-performing strategy A variant $\left(\mathrm{F}_{113} \mathrm{Y}\right)$ with an additional mutation aimed at reducing the steric hindrance of the aliphatic side-chain at position 114 (L114I, since a switch from $\mathrm{F}$ to A in strategy B variants proved less efficient than $\mathrm{F}$ to I). Even though the resulting F113Y-L114I variant proved successful in improving only a few of the conversions seen with F113Y (e.g. 1g, where the increased active site volume better accommodates the $m-\mathrm{Cl}$ substituent), this double variant afforded the highest conversion across the panel for the challenging substrate 1 (18\%).

These results highlight the difficulty in engineering a broadly applicable PAL biocatalyst able to accept diverse highly electron-rich substrates. Even though expanding the hydrophobic binding pocket afforded increased conversions with many of the substrates tested, no additional improvement was seen by broadening the cavity further (as demonstrated by the double variants F113I-L114A and F113I-L114V).

Employing our rational design process, we were able to extend the substrate scope of PbPAL towards very demanding electron-rich substrates. The engineering success with respect to improved acceptance of $\mathbf{1 c}$ and $\mathbf{1 m}$, combined with the existing high efficiency for the respective isomers $\mathbf{1 b}$ and $\mathbf{1}$, suggests that the improvements were mainly achieved through the removal of steric hindrance around the para-position of the substrates. Additionally, the conversion of $\mathbf{1} \mathbf{l}$ and $\mathbf{1 m}$ but poor efficiency with the similarly substituted in and ip imply that the electron-donating potential of methylenedioxy- vs. dimethoxy-substitution is likely lower. This may be due to the constrained 5-membered ring system which contains the two oxygens in the latter case, contravening optimal overlap of lone pairs with the $\pi$-orbitals of the aryl ring system. The clear preferences observed for certain variants towards specific substrates highlight the flexibility of these engineered biocatalysts, diversifying the PAL toolbox.

In addition, with some of the variants we detected very small amounts of the corresponding $\beta$-amino acid as a side product. This is a side activity of PALs we have reported previously, ${ }^{10}$ and that could be mitigated by incubating the biotransformation for shorter times (12-16 h). Longer incubation times (48 h) afforded higher yields of the side product (Supporting Information, Table $\mathrm{S}$ ). The production of the $\beta$-regioisomer is presumably enhanced by the electronic properties of the substrates in this study, as seen with AvPAL ${ }^{10}$ and related aminomutases. ${ }^{11}$

Lastly, to demonstrate the synthetic utility of these enzymes, we performed several preparative scale biotransformations with the best variant for each substrate (giving the optimal trade-off between high conversion and low $\beta$ amino acid side-product formation). Reactions were conducted on a $100 \mathrm{mg}$ scale, affording the corresponding amino acids in excellent conversions and good isolated yield (Scheme $\mathbf{2}$ ), except for $\mathbf{2 h}$, which consistently gave considerable losses during purification. Most of these building blocks have been employed in the synthesis of a wide range of APIs and natural products, such as L-DOPA (from $\mathbf{2 m}$ ), cryptophycin A (from 2g), ${ }^{12}$ anticancer bouvardin derivatives (from $\mathbf{2 f})^{13}$ and fluoroepinephrine (from 2q). ${ }^{14}$

In conclusion, we have successfully engineered a recently discovered PAL to accept electronically and sterically demanding substrates. By using tailored variants, good isolated yields and excellent ee could be demonstrated on preparative scale. Furthermore, this work highlighted the importance of implementing enzyme discovery (starting with a unique template) to facilitate engineering strategies. 


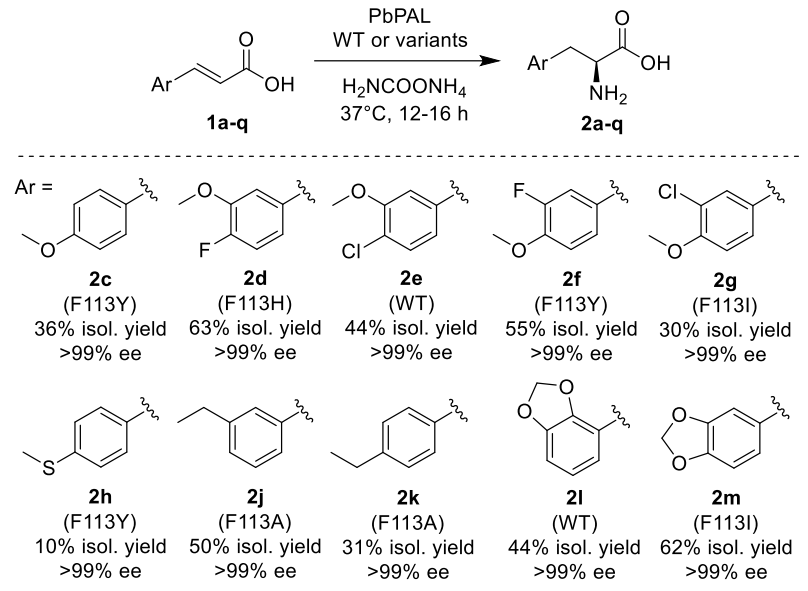

Scheme 2. Preparative scale synthesis of electron-rich phenylalanines.

\section{ASSOCIATED CONTENT}

Supporting Information. Experimental methods, compound characterization data, supporting figures and tables. This material is available free of charge via the Internet at http://pubs.acs.org.

\section{AUTHOR INFORMATION}

\section{Corresponding Author}

* E-mail: nicholas.turner@manchester.ac.uk

\section{Funding Sources}

This work was funded by the Biotechnology and Biological Sciences Research Council (BBSRC) and Glaxo-SmithKline (GSK) under the Strategic Longer and Larger (sLoLa) grant initiative ref. BB/Koo199X/1.

\section{ACKNOWLEDGMENT}

Ulrike Klemstein is kindly acknowledged for assistance with mutagenesis and cloning.

\section{REFERENCES}

[1] a) Parmeggiani, F.; Weise, N. J.; Ahmed, S. T.; Turner, N. J., Synthetic and Therapeutic Applications of Ammonia-lyases and Aminomutases. Chem. Rev. 2018, 118, 73-118; b) Heberling, M. W.; Wu, B.; Bartsch, S.; Janssen, D. B., Priming Ammonia Lyases and Aminomutases for Industrial and Therapeutic Applications. Curr. Opin. Chem. Biol. 2013, 17, 250-260. c) Poppe, L., Methylidene-imidazolone: a Novel Electrophile for Substrate Activation. Curr. Opin. Chem. Biol. 20o1, 5, 512524 .

[2] a) Gloge, A.; Zoń, J.; Kövári, Á.; Poppe, L.; Rétey, J., Phenylalanine Ammonia-lyase: the Use of its Broad Substrate Specificity for Mechanistic Investigations and Biocatalysis - Synthesis of L-Arylalanines. Chem. Eur. J. 20oo, 6, 3386-339o b) Paizs, C.; Toşa, M. I.; Bencze, L. C.; Brem, J.; Irimie, F. D.; Rétey, J., 2-Amino-3-(5-phenylfuran-2-yl)propionic Acids and 5Phenylfuran-2-ylacrylic Acids are Novel Substrates of Phenylalanine Ammonia-Lyase. Heterocycles 2010, 82, 1217-1228; c) Ahmed, S. T.; Parmeggiani, F.; Weise, N. J.; Flitsch, S. L.; Turner, N. J., Synthesis of Enantiomerically Pure Ring-
Substituted L-Pyridylalanines by Biocatalytic Hydroamination. Org. Lett. 2016, 18, 5468-5471.

[3] a) Ahmed, S. T.; Parmeggiani, F.; Weise, N. J.; Flitsch, S. L.; Turner, N. J., Chemoenzymatic Synthesis of Optically Pure Land D-Biarylalanines through Biocatalytic Asymmetric Amination and Palladium-Catalyzed Arylation ACS Catal. 2015, 5, 5410-5413; b) Parmeggiani, F.; Ahmed, S. T.; Weise, N. J.; Turner, N. J., Telescopic One-pot Condensationhydroamination Strategy for the Synthesis of Optically Pure L-Phenylalanines from Benzaldehydes. Tetrahedron 2016, 72, 7256-7262; c) Parmeggiani, F.; Lovelock, S. L.; Weise, N. J.; Ahmed, S. T.; Turner, N. J., Synthesis of D- and LPhenylalanine Derivatives by Phenylalanine Ammonia Lyases: A Multienzymatic Cascade Process. Angew. Chem. Int. Ed. 2015, 54, 4608-4611.

[4] Weise, N. J.; Ahmed, S. T.; Parmeggiani, F.; Galman, J. L.; Charnock, S. L.; Turner, N. J., Zymophore Identification Enables the Discovery of Novel Phenylalanine Ammonia Lyase Enzymes. Sci. Rep., 2017, 7, 13691.

[5] Louie, G. V.; Bowman, M. E.; Moffitt, M. C.; Baiga, T. J.; Moore, B. S.; Noel, J. P., Structural Determinants and Modulation of Substrate Specificity in Phenylalanine-tyrosine Ammonia-lyases. Chem. Biol. 2006, 13, 1327-1338.

[6] Zhu, Y.; Liao, S.; Ye, J.; Zhang, H., Cloning and Characterization of a Novel Tyrosine Ammonia Lyase-encoding Gene Involved in Bagremycins Biosynthesis in Streptomyces sp. Biotechnol. Lett. 2012, 34, 269-274.

[7] Walter, T.; King, Z.; Walker, K. D., A Tyrosine Aminomutase from Rice (Oryza sativa) Isomerizes $(S)$ - $\alpha$ - to $(R)$ - $\beta$-Tyrosine with Unique High Enantioselectivity and Retention of Configuration. Biochemistry 2016, 55, 1-4.

[8] Bencze, L. C.; Filip, A.; Bánoczi, G.; Toşa, M. I.; Irimie, F. D.; Gellért, Á.; Poppe, L.; Paizs, C., Expanding the Substrate Scope of Phenylalanine Ammonia-lyase from Petroselinum crispum towards styrylalanines. Org. Biomol. Chem. 2017, 15, 3717-3727.

[9] Rowles, I; Groenendaal, B.; Binay, B.; Malone, K. J.; Willies, S. C.; Turner, N. J., Engineering of Phenylalanine Ammonia Lyase from Rhodotorula graminis for the Enhanced Synthesis of Unnatural L-Amino Acids. Tetrahedron 2016, 72, 7343-7347.

[10] Weise, N. J.; Parmeggiani, F.; Ahmed, S. T.; Turner, N. J., Discovery and Investigation of Mutase-like Activity in a Phenylalanine Ammonia Lyase from Anabaena variabilis Top. Catal., 2018, DOI: 10.1007/s11244-018-0898-1.

[11] Weise, N. J.; Parmeggiani, F.; Ahmed, S. T.; Turner, N. J., The Bacterial Ammonia Lyase EncP: A Tunable Biocatalyst for the Synthesis of Unnatural Amino Acids. J. Am. Chem. Soc. 2015, 137, 12977-12983.

[12] Trimurtulu, G.; Ohtani, I.; Patterson, G. M. L.; Moore, R. E.; Corbett, T. H.; Valeriote, F. A.; Demchik, L.; Total Structures of Cryptophycins, Potent Antitumor Depsipeptides from the Blue-Green Alga Nostoc sp. Strain GSV 224. J. Am. Chem. Soc. 1994, 116, 4729-4737.

[13] Gladstone, M. N.; Zhang, G.; Sammakia, T., Bouvardin Derivatives and Therapeutic Uses Thereof. WO pat. 2013/126617 A1, 29 August 2013.

[14] Herbert, B.; Kim, I. H.; Kirk, K. L., Synthesis of 2-Fluoro- and 6-Fluoro-(2S,3R)-(3,4-dihydroxyphenyl)serine as Potential in vivo Precursors of Fluorinated Norepinephrines. J. Org. Chem. 2001, 66, 4892-4897. 


\section{TOC graphic}

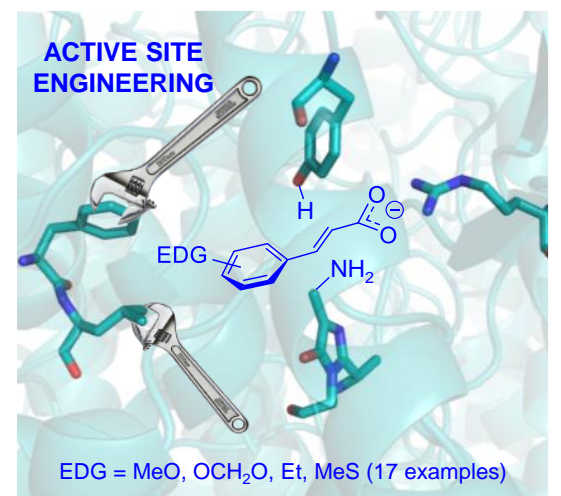

